\title{
On Convergence Analysis and Convergence Enhancement of Power System Least-Squares State Estimators
}

\author{
Robert A.M. van Amerongen, Member IEEE \\ Delft University of Technology, Faculty of Electrical Engineering, Power System Laboratory \\ Mekelweg 4, 2628 CD DELFT, the Netherlands
}

\begin{abstract}
This paper analyses the convergence behavior of the least-squares state estimator. It is shown that the standard algorithm based on the Gauss-Newton method ignores secondorder information. Under some conditions this information is essential for a good convergence. Examples show that an algorithm based on Newton's method reveals a more robust character.

Keywords:

least-squares power-system state-estimation

convergence analysis

Gauss-Newton iteration process
\end{abstract}

\section{INTRODUCTION}

Power System state estimation covers the on-line determination of the state of the system from measurements taken from the real system. To obtain a reliable state, a redundant set of measurements is taken and processed. Since its introduction in the seventies ${ }^{1,2}$, enhancements have been made in the algorithms, in the bad-data analysis, in the observability analysis, and in the topological erroranalysis ${ }^{3}$ :

As far as the algorithms are concerned, each of them can be classified as belonging to either the group of Least-Absolute Value (LAV) estimators or to the group of least-squares estimators. In this paper only the latter category is addressed. In earlier days, the estimator problem was solved by repeatedly solving the normal equations. Later on, a shift to the more robust orthonormal-transformation method took place. The version with the Givens rotation can be regarded as being the most popular one nowadays ${ }^{4}$. There are more variants of the least-squares algorithm, among them are the equality-constrained WLS estimator ${ }^{5}$ and Hachtel's method $^{6}$. The main topic of this paper is relevant to these variants as well.

What all the least-squares estimators have in common is that a cost function that is formed by a sum of (weighted) squared non-linear functions is minimized. The state-estimation problem is thus a

95 WM 215-4 PWRS A paper recommended and approved by the IEET Power System Englneering Committee of the IEEE Power Engineering Society for presentation at the 1995 IBEE/PES Winter Meeting, January 29, to February 2, 1995, New York, NY. Manuscript submitted December 16, 1993; made available for printing November 23, 1994 . non-linear optimization problem. In the standard form there are no additional constraints. In general, a non-linear optimization problem without constraints (NLOP) can be solved by repeatedly solving a quadratic programming problem (QPP), with the Hesse matrix of the cost function of the NLOP acting as the quadratic term ${ }^{7}$. As there are no constraints, the solution of the QPP can be obtained by solving a set of linear equations with the Hesse matrix being the coefficient matrix. Clearly, the same strategy is found when one considers the estimation problem as the solution of a set of nonlinear equations, the Kuhn-Tucker conditions.

Whatever derivation is followed, a Hesse matrix appears which implies that second-order derivatives of the measurement functions must play a role. Standard algorithms, however, do not use any second-order information. Consequently, there is a gap between the algorithms that are derived from optimization theory and the algorithms that are currently used.

This paper's central theme is the exploration of this phenomenon and the consequences of this gap for the convergence speed of the solution process. The paper is organized as follows: in the next section the two versions of the state estimator algorithm are derived. In section III an aid to convergence analysis is provided. In section IV the results of some numerical experiments are presented and discussed. The conclusions close the paper.

\section{BASIC LEAST-SQUARES ESTIMATOR ALGORITHMS}

The model of the power system that is used by the basic least-squares algorithm is given by:

$$
y=f(\underline{x})+\underline{E}
$$

\footnotetext{
where

$y \quad$ is an m-vector with the measurements

$\underline{x}_{t} \quad$ is an $n$-vector with the true state variables

$f$ is the vector that relates measurements and the state variables, the measurement functions

$m$ the number of measurements

$n$ the number of variables, $n=2 N-1$ with $N$ the number of network nodes

$\underline{\epsilon}$ is an m-vector with the measurements noise terms; each term $\epsilon_{i}$ has an expectation $O$ and a deviation $\sigma_{i}$
} 
As the noise terms are known in their statistical quantities only, this model cannot be used to obtain the true state variables. Therefore, a different model is used:

$$
\underline{y}=f(x)+\underline{r}
$$

where

$\underline{x}$ is an $\mathrm{n}$-vector with the variables to be estimated

$\underline{r} \quad$ is an m-vector with the residues

The state $\underline{x}$ is found by solving the unconstrained minimization problem of the form:

$$
J(x)=\sum_{i=1}^{m} w_{i} r_{i}^{2}=\underline{r}^{T} W \underline{r}
$$

where

$J$ the function to be minimized

$W$ a diagonal matrix with the measurements weight factors $w_{i}$, each weight factor is taken as the inverse of the measurements noise variance $\sigma_{i}^{2}$

This problem is solved if the state satisfies the firstorder conditions:

$$
g=A^{T} W \underline{r}=\underline{0}
$$

where

$g \quad$ the gradient of $J$ with respect to $\underline{x}$ (leaving a factor -2 to the side)

$A$ is the $m$ by $n$ Jacobian matrix with the firstorder partial derivatives of the measurement functions $f$ with respect to the variables $\underline{x}$

These conditions constitute a set of non-linear equations. If the set is solved by using Newton's method and if the state dependence of both the residue vector $\underline{r}$ and the Jacobian $A$ is taken into account, the following iteration formula emerges (see appendix A):

$$
G \underline{\Delta x}=\left(A^{T} W A-\sum_{i=1}^{m} w_{i} r_{i} H_{i}\right) \Delta \underline{x}=A^{T} W \underline{r}
$$

where

$G$ is the gain matrix (the Jacobian of $g$ )

$H_{i}$ is the $n$ by $n$ Hesse matrix of the measurement function $f_{i}$

This version is never used in practice. Instead, the following version is used:

$$
G \underline{\Delta x}=\left(A^{T} W A\right) \Delta \underline{x}=A^{T} W \underline{r}
$$

According to standard practice, in the remainder the formulas (5) and (6) are referred to as the Newton and the Gauss-Newton algorithms respectively.

The difference between the two versions is the composition of the gain matrix. In the GaussNewton version the second-order information embedded in the $H_{i}$ matrices is ignored. The circumstances under which this can be problematic can be easily seen:

- if the residue is large due to an error that dominantly propagates in its own residue

- if the measurement function has a relatively high degree of non-linearity such as ampere magnitude measurements with small values of the current. (Note that the voltage measurements have a Hessian equal to the 0 -matrix.)

(It is not correct to argue that a large weight as such is troublesome, a large weight causes the residue to become small thus letting the product $w_{i} r_{i}$ be a wellbehaved number ${ }^{8}$. A large weight is only troublesome through the $A^{T} W A$ term in the gain matrix.)

Although it seems reasonable to shift from the Gauss-Newton to the Newton method throughout, experiments have shown that an iteration process starting from flat voltage can best start with one or more Gauss-Newton iterations and terminate with strict Newton iterates. This is in accordance with the well-known fact that Newton's method works at its best in the neighborhood of the solution. In the section with numerical results, this issue is treated in more detail.

It is interesting to note that the difference between the two versions is caused by the way in which they are derived. The Gauss-Newton version is found by linearizing the optimization problem (3), whereas the Newton version is found by linearizing the Kuhn-Tucker condition (4). A striking similarity can be found in the OPF algorithms: linearizing the optimization problem leads to LP-based and gradient algorithms, linearizing the KT conditions leads to algorithms based on quadratic programming ${ }^{9}$ or the Newton approach ${ }^{10}$. In the discussions to these papers Monticelli and $\mathrm{Wu}$ have already proposed the use of similar techniques in the problem of state estimation with equality constraints. As far as we know, their remarks seem never to have been followed up.

\section{CONVERGENCE ANALYSIS}

In this section a tool is presented with which the differences between Newton and Gauss-Newton methods can be qualified and the corresponding differences in the convergence speed can be quantified.

The problem is to solve the set (4). Its solution is found once a vector $\underline{x}^{*}$ is obtained such that:

$$
g\left(\underline{x}^{*}\right)=\underline{0}
$$

During an iterative process a sequence of vectors $\underline{x}^{k}$ are generated such that ultimately (7) is satisfied. Convergence is guaranteed if during the process for every iteration it holds that: 


$$
\left\|\underline{x}^{k+1}-\underline{x}^{*}\right\|<\left\|\underline{x}^{k}-\underline{x}^{*}\right\|
$$

To say it in words: convergence is guaranteed if during every iteration the current $\underline{x}^{k}$ comes closer to $x^{*}$ than in the previous iteration. Now let us suppose that there are scalars $\rho^{k}$ such that:

$$
\left\|\underline{x}^{k+1}-\underline{x}^{*}\right\| \leq \rho^{k}\left\|\underline{x}^{k}-\underline{x}^{*}\right\|
$$

Convergence is guaranteed if for every $k \rho^{k}<1$. An upper limit for $\rho^{k}$ is found by the spectral radius (the largest absolute eigenvalue) of the matrix expression:

$$
B\left(\underline{x}^{k}\right)=I-G\left(\underline{x}^{k}\right) G\left(\underline{x}^{*}\right)^{-1}
$$

where

$G\left(\underline{x}^{k}\right)$ the Jacobian matrix of $g$ or an approximation to it that is used at iteration $\mathrm{k}$

$G\left(\underline{x}^{*}\right)$ the Jacobian matrix of $g$ that is evaluated at the final solution $\underline{x}^{*}$, this matrix is the exact Jacobian and thus must be the gain matrix of set (5) including the second-order information

(How this relation can be derived is indicated in appendix B.)

Clearly, the sequence $\rho^{k}$ describes the rate of convergence. If these values are small, the rate is high. If the values approach 1 , the rate is very small. If the Newton process uses a constant Jacobian, the $\rho$ has a constant value during the process. If during the process the Jacobian is reevaluated the $\rho$ will change and there are several possibilities as far as the course of $\rho$ is concerned.

Suppose that the Newton process is used, the matrix $G\left(\underline{x}^{k}\right)$ will approach $G\left(\underline{x}^{*}\right)$ and thus $\rho$ approaches to zero at the end of the iteration process.

If, on the other hand, the Gauss-Newton process is used the matrix $G\left(\underline{x}^{k}\right)$ will remain different from $G\left(\underline{x}^{*}\right)$ and thus $\rho$ will approach to a constant nonzero at the end of the iteration process. Note that this constant value, indicated by $\rho^{*}$, is a degree for the convergence speed that is lost when using the Gauss-Newton method. Consequently, it is used throughout in the next section.

\section{NUMERICAL RESULTS}

To qualify and to quantify the difference between the Gauss-Newton and the Newton algorithms, a prototype program for the Newton method has been written by changing an existing Gauss-Newton program based on the normal equations. The additions were formed merely by adding secondorder derivatives to the gain matrix, and for these activities standard modules were taken from an existing Newton-based optimal power flow program.

A number of experiments were conducted. For all these tests a flat voltage was used as the start voltage. The iteration process was terminated as soon as the largest element of the solution was less than $1.0 \mathrm{~d}-07$.

The results of the first tests are presented to

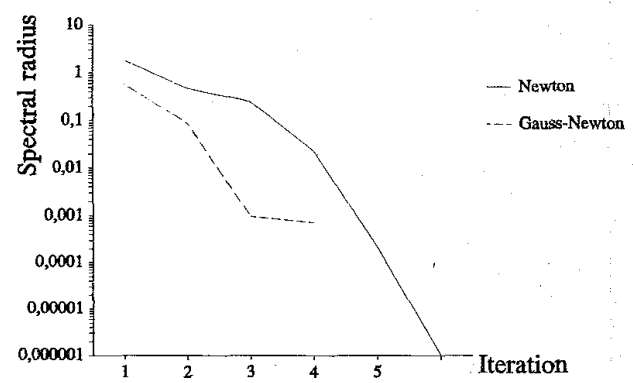

Fig. I Spectral radius in the first test.

show some very basic observations. An 8-node network was used with a measurement pattern based on both line and injection measurements. If there are no errors in the measurement set, the course of $\rho$ is according figure 1 . The remarkable point in this picture is that the convergence of Newton is relative bad in the first iterations and becomes much better after a few iterations. This phenomenon must be explained as follows: when starting the iterations with a flat voltage, the residuals of the power measurements approach the values of the measurements as the calculated values of power injections are zero. There is a large difference between the initial gain matrix and the final gain matrix. It seems thus reasonable to change the policy in that second-order information is added gradually after one or more iterations. The set (5) thus becomes:

$$
G \Delta \underline{x}^{k}=\left(A^{T} W A-\alpha^{k} \sum_{i=1}^{m} w_{i} r_{i} H_{i}\right) \Delta \underline{x}^{k}=A^{T} W \underline{r}
$$

The contribution of the second-order information is governed by a scalar $0 \leq \alpha^{k} \leq 1$. A rule for the determination of its value must be supplicd. As an experiment, the rule was used that in the first iteration no information is added $\left(\alpha^{I}=0\right)$, in the second iteration only $50 \%\left(\alpha^{2}=0.5\right)$ and in the third and subsequent iteration the full $100 \%$ information $(\alpha=1$.$) . With this policy, the behavior of the$ spectral radius is as given in figure 2 .

The course of the spectral radius is now different. It starts lower and has, after a few iterations, the same slope as in test 1 .

In the test so far there was no error in the measurement set. As an demonstration of the basic 


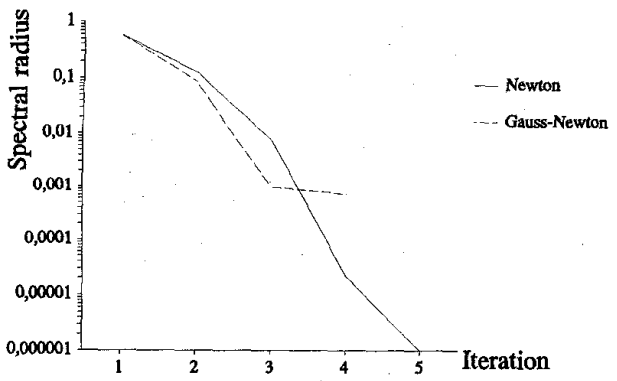

Fig. 2 The spectral radius in the second test.

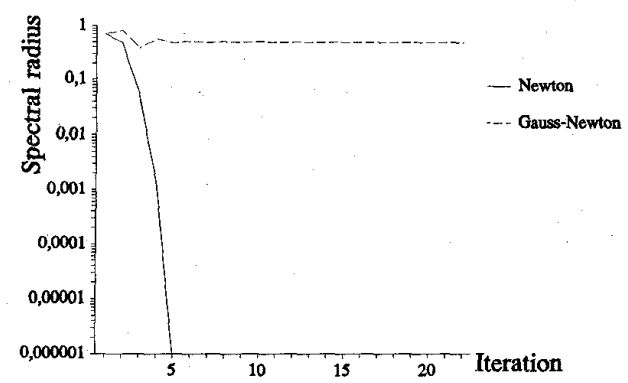

Table I. The number of iterations for the Newton and the GaussNewton method and the spectral radius for the Gauss-Newton method for several values of the error.

\begin{tabular}{||c|r|r|r|}
\hline Error & Newton & $\begin{array}{r}\text { Gauss- } \\
\text { Newton }\end{array}$ & $\rho^{*}$ \\
\hline-5.6 & 6 & 7330 & 0.9982 \\
-5.5 & 6 & 641 & 0.9780 \\
-5.25 & 6 & 196 & 0.9273 \\
-5.0 & 6 & 114 & 0.8760 \\
-4.0 & 6 & 39 & 0.6674 \\
-3.0 & 5 & 20 & 0.4590 \\
-2.0 & 5 & 12 & 0.2639 \\
-1.0 & 5 & 9 & 0.0945 \\
-0.325 & 5 & 5 & 0.0007 \\
0.0 & 5 & 6 & 0.0392 \\
1.0 & 5 & 9 & 0.1444 \\
2.0 & 6 & 12 & 0.2382 \\
3.0 & 6 & 16 & 0.3408 \\
4.0 & 7 & 22 & 0.4873 \\
5.0 & 9 & 97 & 0.8852 \\
\hline
\end{tabular}

Fig. 3 The spectral radius if an error is present.

effects, figure 3 shows the course of the spectral radius if a large error is created. An arbitrary flow measurement was changed from -0.325 to $-3.25 \mathrm{pu}$. The Gauss-Newton method now needs 22 iterations while Newton's method needs only 5 iterations. Note that the $\rho^{*}$ of Gauss-Newton is about 0.5 . This indicates that the distance of the current solution to the final solution reduces by $50 \%$ during the last iteration. Note that figure 3 is representative of a large number of examples: a sharply decreasing spectral radius if Newton's method is used. The Gauss-Newton method, on the other hand, exhibits, after a few iterations, a constant spectral radius. This typically indicates linear convergence.

For several other values of the specific error we conducted such a test. The results are summarized in table I. For very extreme values of the error the Gauss-Newton requires a large number of iterations. In those cases $\rho^{*}$, the spectral radius for the GaussNewton method evaluated at the final solution, approaches 1 . The Newton method requires only a small number of iterations.

Although the differences presented in table I are remarkable, they may not be used to promote the Newton method as the corresponding physical situations are not really reasonable. In general, it was observed that estimation problems with realistic single or multiple errors in the measurement sets

can be solved as effectively by using the GaussNewton method as by the Newton method.

Things went drastically different if topology errors are simulated. In test 4 the IEEE 30 node network was used. A measurement pattern was designed where all the nodal injection powers and all the lines powers were measured. Beside, the voltages of the PV nodes were added. For every branch (excluding those whose removal caused islanding) the estimation was performed with both Gauss-Newton and Newton. The measurements were simulated with the branch in service while the estimator algorithm assumes the particular branch to be out of service. The flow measurements in that branch were not taken into account. The scalars $\alpha$ where set as follows: for the first three iteration $\alpha=0$ (thus Gauss-Newton is in effect), $\alpha^{4}=0.25$, $\alpha^{5}=0.50, \alpha^{6}=0.75$ and the pure Newton is in effect from the $7^{\text {th }}$ iteration. The results are given in table II. Here, again, $\rho^{*}$ is the spectral radius for the Gauss-Newton method evaluated at the final solution.

For the cases where convergence is obtained, Newton was nearly always better. There are, however, a few cases where both Newton and Gauss-Newton diverged or failed to produce a realizable solution.

Similar experiments were conducted making use of the network of one of the Dutch utilities. These tests did not produce any additional insight with one exception: in one case Gauss-Newton diverged and 
Table II. The number of iterations for the Newton and the GaussNewton method and the spectral radius for the Gauss-Newton method in the case of topology errors (branches).

d a diverging case

f a failure case

\begin{tabular}{|c|c|c|c|}
\hline Branch & Newton & $\begin{array}{c}\text { Gauss- } \\
\text { Newton }\end{array}$ & $\rho^{*}$ \\
\hline 1 & 9 & 14 & 0.188 \\
\hline 2 & 8 & 14 & 0.267 \\
\hline 3 & 8 & 12 & 0.265 \\
\hline 4 & 11 & 14 & 0.185 \\
\hline 5 & 8 & 12 & 0.272 \\
\hline 6 & 8 & 9 & 0.132 \\
\hline 7 & 8 & 29 & 0.571 \\
\hline 8 & 8 & 15 & 0.313 \\
\hline 9 & 9 & 139 & 0.900 \\
\hline 10 & 9 & 25 & 0.495 \\
\hline 11 & 9 & 21 & 0.474 \\
\hline 12 & 6 & 6 & 0.061 \\
\hline 14 & $\mathrm{~d}$ & $\mathrm{~d}$ & \\
\hline 15 & 8 & 11 & 0.223 \\
\hline 17 & 10 & 11 & 0.091 \\
\hline 18 & $\mathrm{f}$ & $\mathrm{f}$ & \\
\hline 19 & 9 & 12 & 0.172 \\
\hline 20 & 8 & 12 & 0.243 \\
\hline 21 & 9 & 17 & 0.372 \\
\hline 22 & 13 & 16 & 0.358 \\
\hline 23 & 8 & 12 & 0.178 \\
\hline 24 & 8 & 22 & 0.574 \\
\hline 25 & 9 & 49 & 0.739 \\
\hline 26 & 8 & 18 & 0.436 \\
\hline 27 & d & $\mathrm{f}$ & \\
\hline 28 & 10 & 42 & 0.735 \\
\hline 29 & 9 & 15 & 0.310 \\
\hline 30 & 8 & 11 & 0.182 \\
\hline 31 & d & $\mathrm{f}$ & \\
\hline 32 & 9 & 18 & 0.416 \\
\hline 33 & $\mathrm{f}$ & $\mathrm{f}$ & \\
\hline 35 & $\mathrm{f}$ & $\mathrm{f}$ & \\
\hline 36 & 9 & 23 & 0.507 \\
\hline 37 & 8 & 22 & 0.533 \\
\hline 38 & 9 . & 17 & 0.332 \\
\hline 39 & 9 & 20 & 0.362 \\
\hline 40 & 8 & 14 & 0.310 \\
\hline 41 & $\mathrm{~d}$ & f & \\
\hline
\end{tabular}

Newton converged quickly.

Table III. Comparison of CPU times.

\begin{tabular}{|c|r|r|}
\hline CPU times & Newton & $\begin{array}{r}\text { Gauss- } \\
\text { Newton }\end{array}$ \\
\hline in seconds & 4.67 & 4.12 \\
\hline normalized & 1.13 & 1.00 \\
\hline
\end{tabular}

Finally, we did a test to compare the CPU time of both the methods. The computer is a $66 \mathrm{MHz}$ 486 PC. The IEEE 118-node network was used. Both methods needed 5 iterations to solve the problem. The CPU times (for the complete solution process) are given in table III. For the test example an increase of the CPU time of $13 \%$ was found. As the number of iterations with Newton usually is (much) smaller, the increase of the CPU-time was by far outweighed by the decrease in the number of iterations.

\section{DISCUSSION AND CONCLUSIONS}

In this paper it is shown that a correct derivation of the state-estimator iteration algorithm produces a gain matrix with second-order derivatives of the measurement functions. This version is called the Newton method. If these second-order derivatives are omitted from the gain matrix, the well-known iteration formula according to the Gauss-Newton method appears.

Simulation shows that if only one or more simple errors are in the measurement set, there is no need to replace the Gauss-Newton by the Newton method. Complicated errors such as topology errors, can devastate the convergence of the GaussNewton method and a shift to Newton must strongly be considered.

Simulation also shows that in the first few iterations the second-order information must be ignored. To say it in other words: start with GaussNewton and shift gradually to Newton. The exogenously determined fixed rule that is used, however, must definitely be replaced by an endogenously determined rule. To say it in other words: $\alpha^{k}$ must be made dependent on the course of the solution process. Future research is conducted along two lines: first, how can converging GaussNewton cases be accelerated by using Newton's method; second, how can diverging Gauss-Newton cases be detected and, if possible, made converging by Newton's method.

\section{ACKNOWLEDGEMENTS}

The author wish to express his gratitude to Nelis Bijl of the Power System Laboratory who familiarized him with the convergence analysis technique used in this paper, and to $\mathrm{Mr}$. $\mathrm{P}$. Sonneveld of the Department of Mathematics for his productive discussions and helpful suggestions.

\section{REFERENCES}

1 F.C. Schweppe, J. Wildes: "Power System Static-State Estimation, Part I: Exact Model", IEEE Transactions on Power Apparatus and 
Systems, vol. PAS-89, pp. 120-125, 1970

2 R.E. Larson, W.F. Tinney, J. Peschon: "State Estimation in Power Systems, Part I: Theory and Feasibility", IEEE Transactions on Power Apparatus and Systems, vol. PAS-89, pp. 345352, 1970

3 M.B. Do Coutto Filho, A.M. Leite da Silva, D.M. Falcão: "Bibliography on Power System State Estimation (1968-1989)", IEEE Transactions on Power Systems, vol. 5, pp. 950 961, 1990

4 N. Vempati, I.W. Slutsker, W.F. Tinney: "Enhancements to Givens Rotations for Power System State Estimation", IEEE Transactions on Power Systems, vol. 6, pp. 842-849, 1991

5 F.C. Aschmoneit, N.M. Peterson, E.C. Adrian: "State Estimation with Equality Constraints", 1977 PICA Conference Proceedings, pp. 427430

6 A. Gjelsvik, S. Aam, L. Holten: "Hachtel's Augmented Matrix Method - A Rapid Method Improving Numerical Stability in Power System Static State Estimation", IEEE Transactions on Power Apparatus and Systems, vol. PAS-104, pp. 2987-2993, 1985

7 P.E. Gill, W. Murray, M.H. Wright: Practical Optimization, London: Academic Press, 1981

8 R.A.M. van Ámerongen: "On the Exact Incorporation of Virtual Measurements in Orthogonal-Transformation Based StateEstimation Procedures", Electrical Power \& Energy Systems, vol. 13, pp. 167-174, 1991

9 R.C. Burchett, H.H. Happ, D.R. Vierath: "Quadratically Convergent Optimal Power Flow", IEEE Transactions on Power Apparatus and Systems, vol. PAS-103, pp. 3267-3275, 1984

10 D.I. Sun, B. Ashly, B. Brewer, A. Hughes, W.F. Tinney: "Optimal Power Flow by Newton Approach", IEEE Transactions on Power Apparatus and Systems, vol. PAS-103, pp. 2864-2880, 1984

11 J.M. Ortega: Numerical Analysis - A Second Course, Philadelphia: Siam, 1990

12 J.M. Ortega, W.C. Rheinboldt: Iterative Solution of Non-Linear Equations in Several Variables, New York: Academic Press, 1970

\section{APPENDIX A}

In this appendix equation (5) is derived and some additional information on the implementation of the Newton method is offered.

The problem (3) is to minimize:

$$
J(\underline{x})=\sum_{i=1}^{m} w_{i} r_{i}^{2}
$$

where each residue is given by (see (2)):

$$
r_{i}=y_{i}-f_{i}(x)
$$

The minimum of (A.1) is found if:

$$
J_{x}(\underline{x})=\underline{0}
$$

where

$J_{x}(\underline{x}) \quad$ is the gradient of $J$ with respect to the variable vector $\underline{x}$.

Now let us suppose that (A.3) does not hold. Then a $\Delta \underline{x}$ must be found such that:

$$
J_{x}(\underline{x}+\underline{\Delta x})=\underline{0}
$$

A linearization of this equation yields:

$$
J_{x x}(\underline{x}) \underline{\Delta x}=-J_{x}(\underline{x})
$$

where

$J_{x x}(\underline{x})$ is the Hesse matrix (the matrix with second-order partial derivatives) of the function $J$.

The element expression and the matrix expression of the gradient $J_{x}(\underline{x})$ are given by:

$$
\begin{gathered}
\frac{\partial J}{\partial x_{j}}=2 \sum_{i=1}^{m} w_{i} r_{i} \frac{\partial r_{i}}{\partial x_{j}}=-2 \sum_{i=1}^{m} w_{i} r_{i} \frac{\partial f_{i}}{\partial x_{j}} \\
J_{x}(\underline{x})=-2 A^{T} W \underline{r}
\end{gathered}
$$

In the latter $A$ is the Jacobian matrix of the measurement functions.

The element expression and the matrix expression of the Hesse matrix $J_{x x}(\underline{x})$ are given by:

$$
\begin{gathered}
\frac{\partial^{2} J}{\partial x_{j} \partial x_{k}}=2 \sum_{i=1}^{m} w_{i} \frac{\partial f_{i}}{\partial x_{j}} \frac{\partial f_{i}}{\partial x_{k}}-2 \sum_{i=1}^{m} w_{i} r_{i} \frac{\partial^{2} f_{i}}{\partial x_{j} \partial x_{k}} \\
J_{x x}(x)=2 A^{T} W A-2 \sum_{i=1}^{m} w_{i} r_{i} H_{i}
\end{gathered}
$$

where $H_{i}$ is the Hesse matrix of measurement function $f_{i}$.

The basis equation of the Newton method (5) is found by substituting (A.7) and (A.9) into (A.5) while dropping the scalar 2 :

$$
\left(A^{T} W A-\sum_{i=1}^{m} w_{i} r_{i} H_{i}\right) \Delta \underline{x}=A^{T} W \underline{r}
$$

Regarding the implementation of the Newton method, the following issues are relevant. If we compare the Newton method with the Gauss-Newton method, it needs no explanation that only the gain matrix undergoes any change in that the terms with the second-order derivatives are added. In general, every measurement adds to it with: 


$$
w_{i} r_{i} H_{i}=w_{i} r_{i}\left[\frac{\partial^{2} f_{i}}{\partial x_{j} \partial x_{k}}\right]
$$

The specific contribution depends on the type of the measurement and in this regard the following applies:

- for a nodal voltage measurement the Hesse matrix is zero because the function is a linear one in $\underline{x}$;

- for each line flow measurement there are 16 non-zero elements in $H_{i}$; these elements can be found by differentiating the relevant power flow equation of a single branch;

- as an injection flow can be regarded as the sum of the branch flows, the Hesse matrix of an injection flow measurement can be composed of the separate Hesse matrices from these branch flows.

\section{APPENDIX B}

In this appendix the derivation of some of the results used in section III are given. In the derivation the work of Ortega $a^{11,12}$ is closely followed.

At the center of the problem is the solution of the set of non-linear equations (4)

$$
g(x)=\underline{0}
$$

by an iterative process. An example is Newton's iteration process where repeatedly the set

$$
G \Delta \underline{x}=-g(\underline{x})
$$

is solved. The iteration scheme thus is:

$$
\underline{x}^{k+1}=\underline{x}^{k}-G-1
$$

This process is denoted by:

$$
\underline{x}^{k+1}=\underline{b}\left(\underline{x}^{k}\right)
$$

for $\mathrm{k}=0,1,2, \ldots$

A solution $\underline{x}^{*}=\underline{b}\left(x^{*}\right)$ of (B.4) is called a fixed point. Before using Ostrowski's theorem, we use the following definition:

a fixed point $x^{*}$ is a point of attraction of the iteration (B.4) (alternatively, one says that the iteration is locally convergent at $\left.\underline{x}^{*}\right)$ if there is an open neighborhood $\mathrm{S}$ of $\underline{x}^{*}$ such that whenever $x^{0} \in S$, the iterates (B.4) are well-defined and converge to $\underline{x}^{*}$.

A local convergence theorem is given by Ostrowski's Theorem: assume that $\underline{b}$ of (B.4) is differentiable at the fixed point $\underline{x}^{*}$ and that it holds that

$$
\rho=\xi\left(B\left(x^{*}\right)\right)<1
$$

then $\underline{x}^{*}$ is a point of attraction. In this formulation the following notation is used:

$B\left(x^{*}\right)$ the Jacobian matrix of $\underline{b}$ evaluated at the fixed point $\underline{x}^{*}$

$\xi$ the spectral radius (the largest eigenvalue) of $B$

We briefly summarize the proof. For a sufficiently small $\epsilon>0$ there is a norm on B such that it holds that

$$
\left\|B\left(\underline{x}^{*}\right)\right\| \leq \rho+\epsilon
$$

As $\underline{b}$ is differentiable, there is a $\delta>0$ so that if

$$
S \equiv\left\{\underline{x}:\left\|\underline{x}-\underline{x}^{*}\right\|<\delta\right\}
$$

then it holds:

$$
\left\|\underline{b}(x)-\underline{b}\left(\underline{x}^{*}\right)-B\left(\underline{x}^{*}\right)\left(\underline{x}-\underline{x}^{*}\right)\right\| \leq \epsilon\left\|\underline{x}-\underline{x}^{*}\right\|
$$

whenever $x \in S$. The following general inequality holds:

$$
\begin{gathered}
\left\|\underline{b}(x)-\underline{b}\left(\underline{x}^{*}\right)\right\| \leq \\
\left\|\underline{b}(\underline{x})-\underline{b}\left(\underline{x}^{*}\right)-B\left(\underline{x}^{*}\right)\left(\underline{x}-\underline{x}^{*}\right)\right\|+\left\|B\left(\underline{x}^{*}\right)\left(\underline{x}-\underline{x}^{*}\right)\right\|
\end{gathered}
$$

Incorporating (B.8) into (B.9) yields:

$$
\left\|\underline{b}(x)-\underline{b}\left(\underline{x}^{*}\right)\right\| \leq \epsilon\left\|\underline{x}-\underline{x}^{*}\right\|+\left\|B\left(\underline{x}^{*}\right)\left(\underline{x}-\underline{x}^{*}\right)\right\|
$$

and incorporating (B.6) into (B.10) gives:

$$
\left\|\underline{b}(x)-\underline{b}\left(\underline{x}^{*}\right)\right\| \leq(\rho+2 \epsilon)\left\|\underline{x}-\underline{x}^{*}\right\|
$$

Since $\rho<1$, we may assume that $\epsilon>0$ is chosen so that $\rho^{\prime}=\rho+2 \epsilon<1$. Therefore, at iteration $\mathrm{k}$, the basic result emerges:

$$
\left\|\underline{x}^{k+1}-\underline{x}^{*}\right\| \leq \rho^{\prime}\left\|\underline{x}^{k}-\underline{x}^{*}\right\|
$$

Two observations hold:

- if $\rho<1$ then $\rho^{\prime}<1$ and convergence is guaranteed

- a measure for the convergence speed is given by $\rho$

In this paper we approximate the convergence speed by $\rho$.

Robert A.M. van Amerongen (M $M^{\circ} 8$ ) was born in Heemstede the Netherlands, on May 3, 1950. He studied electrical engineering at the Delft University of Technology, and economics at the Erasmus University, Rotterdam and the University of Amsterdam. He received his M.Sc. in Electrical Engineering in 1978 and his Master's in Economics in 1987. In 1978 he entered the Power System Laboratory of the Delft University of Technology as a research assistant where he is now responsible for education and research. His main areas of interest are electric power-system analysis, including network calculation and its applications, optimization and estimation. During 1992 he was seconded to the Power System Operations and Control Department of Sep (Dutch Electricity Generating Board) where he was involved in the design of a unit-commitment procedure for combined electricity and heat production. 